\title{
Assessment of drug-eluting stents and bioresorbable stents by grayscale IVUS and IVUS-based imaging modalities
}

\author{
Salvatore Brugaletta $\cdot$ Jose Ribamar Costa Jr • \\ Hector M. Garcia-Garcia
}

Received: 7 December 2010/Accepted: 30 December 2010/Published online: 30 January 2011

(C) Springer Science+Business Media, B.V. 2011

\begin{abstract}
Grayscale IVUS and IVUS-based imaging modalities during the last years have become useful in the assessment not only of drug eluting stent, but also of new bioresorbable vascular scaffolds. Although IVUS resolution is not sufficient for determining stent coverage (optical coherence tomography is the gold standard), serial IVUS can measure intimal hyperplasia, assess acute and late incomplete stent apposition, detect the presence and persistence of edge dissections, study edge effects and look for causes of restenosis and thrombosis. In addition other IVUS-based imaging modalities, such as IVUS-VH, iMAP or palpography, can be used to study the serial compositional and mechanical changes of the plaque behind stent struts and also to follow the bioresorption of the new bioresorbable scaffolds, analyzing the backscattering signal coming from the polymeric struts. This review details and evaluates grayscale IVUS and IVUS-based techniques findings in clinical trials, highlighting the usefulness of these imaging modalities in the study of drug eluting stents and bioresorbable vascular scaffold.
\end{abstract}

S. Brugaletta - H. M. Garcia-Garcia $(\bowtie)$

Thoraxcenter, Z120, Erasmus MC, Dr. Molewaterplein

40, 3015 Rotterdam, The Netherlands

e-mail: h.garciagarcia@erasmusmc.nl

J. R. Costa Jr

Instituto Dante Pazzanese, Sao Paulo, Brazil
Keywords Intravascular ultrasound - Stent · Tissue characterization

\section{Introduction}

Intravascular ultrasound (IVUS) is an adjunctive diagnostic technique that provides detailed crosssectional imaging of the coronary arteries. In addition to assisting in the assessment of the morphology and composition of coronary lesions and the appropriate strategy to address them, IVUS can aid in confirming the success of stent deployment and placement [1]. According to the 2005 American College of Cardiology/American Heart Association/Society for Cardiovascular Angiography and Interventions (ACC/ AHA/SCAI) 2005 Guideline Update for PCI, it is reasonable to use IVUS to: (1) assess the adequacy of coronary stent deployment, including the extent of apposition and minimum luminal diameter within the stent; (2) determine the cause of stent restenosis and guide selection of appropriate therapy; (3) evaluate coronary obstruction in a patient with a suspected flow-limiting stenosis when angiography is difficult because of location. These recommendations are made based on a level of evidence that supports the efficacy of IVUS. In addition, IVUS may be used to assess lesion's characteristics and vessel dimensions before PCI to select an optimal revascularization device, although the efficacy of this application is less well established [2]. 
Complementary to grayscale IVUS, tissue characterization by IVUS radiofrequency data, among others, Virtual Histology (VH) [3, 4] analysis has the potential to add valuable information on the pathogenesis of restenosis by providing information on plaque composition [5], on the change in composition of plaque behind the stents [6] and on the quantification of bioresorption process for new bioresorbable vascular scaffolds (BVS) $[7,8]$. At the same time iMAP and palpography are two techniques that interpret the radiofrequency signal for tissue characterization and for strain of the vessel wall. All these techniques are relatively new and their clinical usefulness has still to be elucidated.

This review focuses on the usefulness of IVUS grayscale and IVUS-based imaging modalities in the study of drug-eluting stents (DES) and bioresorbable vascular scaffolds (BVS).

\section{IVUS-guided des implantation}

The use of IVUS imaging is associated with favorable outcomes in patients who received DES implantation during PCI [9-11]. A number of steps are recommended to minimize the risk of adverse events. Before stent deployment, IVUS should be used to confirm that lesion severity warrants DES implantation. It is also important to identify proximal and distal reference sites or "landing zones". To select a DES size and length that will reduce the risk of a proximal or distal edge stenosis, measure vessel size and the distance between the proximal and distal stent edge landing zones, respectively, in order to avoid the longitudinal and axial geographical miss [12]. If necessary, fine-tune the distance so that side branches are not covered. Finally, select an anatomic structure to serve as a fiduciary point linking IVUS imaging and angiography. Post-intervention IVUS should be used to identify and measure the minimum crosssectional area; under-expansion should be addressed with additional inflations at higher pressure (compliance charts of the manufacturers cannot be used to guarantee adequate stent expansion). Proximal and distal stent edges must be analyzed to reveal any secondary uncovered plaques. The final step is to ensure that important complications uncovered by IVUS, such as lumen-compromising edge dissections, intra- and extramural hematomas, and perforations, are treated using appropriate clinical techniques.
Therefore, a number of technical issues are associated with IVUS use in DES implantation, including stent expansion, residual edge stenosis/ dissection, and malapposition.

\section{IVUS assessment of DES expansion}

Lesions that cause stent thrombosis after successful DES deployment are often a consequence of stent under-expansion and residual reference segment stenosis, because inadequate expansion can lead to abnormal shear stress (see Table 1, MUSIC criteria of optimal stent expansion) [13]. For instance, Fujii et al. [14], comparing all patients who received metallic stent eluting sirolimus implantation and in which IVUS was performed during PCI or at the time of stent thrombosis, reported that the minimum stent crosssectional area $\left(4.3 \pm 1.6 \mathrm{~mm}^{2}\right.$ vs. $6.2 \pm 1.9 \mathrm{~mm}^{2}$, $P<0.001)$ and the degree of stent expansion $(0.65 \pm$ 0.18 vs. $0.85 \pm 0.14, P<0.001)$ were significantly smaller in the stent thrombosis group compared with matched controls. A second study, which compared IVUS imaging after DES implantation in 13 patients with matched controls, reported similar findings [15]. DES patients who developed stent thrombosis showed a smaller minimum stent area $\left(4.6 \pm 1.1 \mathrm{~mm}^{2}\right.$ vs. $\left.5.6 \pm 1.7 \mathrm{~mm}^{2}, P<0.0489\right)$ and a larger proximal reference plaque burden $(0.66 \pm 0.08$ vs. $0.56 \pm 0.11$,

Table 1 IVUS definitions

MUSIC criteria of optimal stent expansion:

1. Complete apposition of the stent over its entire length against the vessel wall.

2. In-stent minimal lumen area $\geq 90 \%$ of the average reference lumen area or $\geq 100 \%$ of lumen area of the reference segment with the lowest lumen area. In-stent lumen area of proximal stent entrance $\geq 90 \%$ of proximal reference lumen area. In case the in-stent luminal area $>90$ $\mathrm{mm}^{2}$. In-stent minimal lumen area $\geq 80 \%$ of the average reference lumen area or $\geq 90 \%$ of lumen area of the reference segment with the lowest lumen area. In-stent lumen area of proximal stent entrance $\geq 90 \%$ of proximal reference lumen area.

3. Symmetric stent expansion defined by stent diameter $\mathrm{min} /$ stent diameter $\max \geq 0.7$.

Incomplete stent apposition (ISA): separation of at least 1 stent strut from the intimal wall not overlapping a side branch with evidence of blood speckling (indicating flow):

Early if it is noted after stent implantation

Late-acquired if it was not present on IVUS analysis baseline but developed during the follow-up period during 
$P<0.0018$ ). Thus, ensuring proper stent expansion is essential for the prevention of DES stent thrombosis.

Inadequate stent expansion also increases the likelihood of restenosis. Serial IVUS analysis from the Sirolimus-Eluting Stent in Coronary Lesions (SIRIUS) trial showed that a minimum stent area $<5.0 \mathrm{~mm}^{2}$ was responsible for the majority of SES restenosis in the trial [16]. A study of IVUS predictors of angiographic restenosis in a large series of Korean patients (550 patients with 670 lesions) treated with SES implantation found that the immediate post-procedure IVUS cutoffs that best predicted angiographic restenosis were a minimum stent crosssectional area of $5.5 \mathrm{~mm}^{2}$ and a stent length of $40 \mathrm{~mm}$ [17]. Finally, data from the TAXUS trials identified $6.0 \mathrm{~mm}^{2}$ as the minimum stent area to reduce the risk of revascularization. Examination of sensitivity and specificity curves in these 3 analyses confirmed that the minimum stent area that best separated DES restenosis from no restenosis was between 5.0 and $6.0 \mathrm{~mm}^{2}$, although it is not possible to identify statistically a specific minimum stent cross sectional area value, because in all studies there is a stepwise relation between the final stent cross sectional area and freedom from restenosis. Nevertheless, these studies showed that an optimal stent expansion, especially in complex lesions, was associated with an even lower rate of DES restenosis.

IVUS assessment of residual edge stenoses or dissection

Residual plaque burden also is a predictor of late stent thrombosis. In the retrospective study of metallic stent eluting sirolimus implantation, Fujii et al. reported also that the stent thrombosis group was significantly more likely than control patients to have residual edge stenosis $(67 \%$ vs. $9 \%, P<0.001)$, which was defined as edge lumen cross-sectional area $<4 \mathrm{~mm}^{2}$ and a plaque burden $>70 \%$. [14] Analysis of IVUS parameters in SIRIUS trial patients established a similar relation between longitudinal geographic miss and stent thrombosis. Sakurai et al. examined 317 edges of 167 stents that had 18 edge stenoses after 8 months [18]. Patients with peri-stent stenosis had a significantly higher maximum reference plaque area compared to patients without peristent stenosis $(60.5 \% \pm 9.0$ vs. $48.8 \% \pm 11.5$, $P<0.030)$. These studies propose that longitudinal geographic miss is associated with target lesions revascularization.

It is noteworthy to consider also that the serial geometrical changes at the edges of a stent vary depending on the location (proximal vs. distal edge), the shear stress conditions [19], the morphological baseline characteristics of the segment (degree of obstruction) and baseline tissue composition. In TAXUS II [20], positive remodeling was observed only at the distal edges in both the slow and moderate release Taxus groups. This was almost entirely due to an increase in plaque size and there was hardly any change in lumen size. Meanwhile, in the TAXUS IV study [21], almost no change was observed in vessel size at the proximal edge and a trend towards negative remodeling was observed at the distal edge. It is difficult to put these two studies into perspective; perhaps due to differences in sample size and potential selection bias acknowledged by the authors of TAXUS IV. In the ENDEAVOR II study [22], IVUS analysis at 8 months found the minimum luminal area in the distal edge of the metallic stent eluting zotarolimus (ZES) to be significantly higher than that of the bare metal stents (BMS). In detailed sub-segmental analyses, loss of the mean luminal area did not differ between the two stent groups at the proximal edges. Conversely, at the first millimeter of the distal edge, the mean plaque area increased more with the BMS than the ZES, while the mean vessel area was not different between the two groups. This resulted in a significantly smaller mean luminal area in the ZES than the BMS group.

\section{IVUS evaluation of DES apposition}

Incomplete stent apposition (ISA) (Table 1) has been postulated to be one of the potential causes of stent thrombosis after DES implantation. However, there are no good data linking isolated acute stent malapposition to adverse clinical events. The reported prevalence of ISA post-stenting among DES studies varies from 2.4 to $34.4 \%$ [21, 23-27]. On the other hand, although late-acquired ISA has, by definition, the same IVUS appearance as post-stenting ISA, its postulated mechanisms are completely different including progressive expansion of the vessel wall, thrombus resolution and lack of tissue growth around the stent struts [27]. In the literature, the incidence ranges from 0.0 to $16.7 \%$ [17, 21, 23, 24, 26-29]. Of 
note, no clinical events related to ISA have been reported in these trials. In other words, stent sizing and residual disease are the critical parameters associated with adverse outcomes.

Investigating the fate of incomplete stent apposition in 168 consecutive patients with 182 lesions who received SES, Kimura et al. found no clinical, procedural, or IVUS measurement difference between patients and lesions with isolated stent malapposition and those without, although individuals with persistent incomplete stent apposition were more likely to have evidence of superficial lesion calcium [26]. Most cases of acute stent malapposition resolved over time in this study; those that did not, had no effect on the incidence of stent thrombosis or in-stent restenosis. Similar results were reported by Hong et al. who examined 557 patients with 705 native lesions who received DES implantation with IVUS imaging [27]. Here, again, late stent malapposition (at mean follow-up of 10.2 months) was not associated with any major adverse cardiac events. More recently, Cook et al. reported 11 patients with very late DES thromboses and compared them with 198 controls that had undergone routine IVUS follow-up but did not develop stent thrombosis: incomplete stent apposition was present at the time of thrombosis in $77 \%$ of patients with very late DES thrombosis vs. $12 \%$ of controls $(P<0.0001)$ [30]. Very late DES thrombosis has been also associated with histopathological and serological signs of inflammation, in particular with eosinophil infiltration: the ISA was associated with total eosinophil count [30].

\section{IVUS and DES-restenosis}

The MUSIC study [13] assessed the impact of IVUSguided stent implantation on the 6 months restenosis rate. In this study, complete stent apposition, well expansion (in-stent minimal lumen area $>90 \%$ of the average reference lumen area) and symmetric stent expansion defined by minimum lumen diameter/ maximum lumen diameter $\geq 0.7$ were considered for assessing optimal stent deployment (Table 1). This study demonstrated that satisfactory clinical and angiographic outcome at 6 months can be achieved by using IVUS guidance. It has already been discussed above how an under-expansion of the stent (minimum luminal cross sectional area between 5.0 and $6.0 \mathrm{~mm}^{2}$ ) may play an important role in stent failure [16, 17]. Unfortunately, optimal minimum stent area values are not frequently achieved in current practice. In a study that enrolled 200 patients with de novo coronary lesions treated with metallic stents eluting either sirolimus ( $n=133$ patients) or paclitaxel ( $n=67$ patients) under IVUS guidance, $24 \%$ of sirolimus-eluting stent and $28 \%$ of paclitaxeleluting stent did not achieve a final minimum stent area of $5 \mathrm{~mm}^{2}$ [31].

Another reported cause of stent restenosis, potentially valuable by IVUS, is stent fracture. At two US centers 3,920 patients in a period of 1 year were treated with drug-eluting stents, and in-stent restenosis was reported in 188 patients (121 sirolimuseluting stent and 67 paclitaxel-eluting stent). In 35 patients out of $188(18.6 \%)$ stent fracture was observed. Only cases with severe stent fracture (complete separation of stent segments) were included and mild (single-strut fracture) and moderate (more than one strut fracture, but without clear separation of fractured stent segments) stent fractures were excluded. In other words, it appears that in onefifth of the cases of in-stent restenosis, stent fracture is the main mechanism of restenosis. The presence of vessel tortuosity, use of SES and overlapping stents were the factors more frequently associated with stent fracture, while larger stent diameter was protective. According to the authors of this study, high magnification cine-angiography is the best method to diagnose stent fracture and, although intravascular ultrasound was performed in 10 out of the 35 cases of stent fracture, it did not add any value to high magnification cine-angiography [32]. However, in a most recent article studying 83 lesions in 56 patients by serial angiography and IVUS at baseline and at 6 months follow-up, Yamada et al. found that at 6 months by angiography no cases of stent fracture were detected, while by IVUS 3 cases were detected [33]. Moreover, IVUS is not only helpful to identify stent fracture as cause of stent failure, but more importantly to understand possible mechanisms of stent fractures, such as aneurysm formation [34].

\section{IVUS virtual histology}

Regarding the vascular responses after stenting (i.e. restenosis and remodeling), a response-to-injury 
pattern of wound healing occurs, mainly characterized by an increase in smooth muscle cells and extracellular matrix [35]. This depends on the drug type and the release kinetics. The most commonly used DES, sirolimus- and paclitaxel eluting stents, reduce restenosis by interfering with muscle cell proliferation through different modes of action [36]. In this way, tissue characterization pre and poststenting with IVUS-VH is an important tool to define plaque type pre-stent implantation and its temporal modifications. VH (EagleEye ${ }^{\mathrm{TM}}$; Volcano Corporation, Rancho Cordova, CA, USA) uses an electronic catheter that acquires ECG gated data for tissue characterization. In addition, $\mathrm{VH}$ feeds the spectra that are obtained from the radiofrequency data using autoregressive models into a classification tree that has reported diagnostic accuracies of over $90 \%$ for each plaque component as compared to histology [4] Four tissue components (necrotic core-red; dense calcium-white; fibrous-green; and fibrofattylight green) were identified with this autoregressive classification system [3, 37].

One of the proposed pathological mechanisms of coronary stent thrombosis is stenting of necrotic core rich plaques with extensive tissue prolapse and plaque disruption in the proximity of the stented arterial segment [38-40]. Thus, pre-stenting imaging using IVUS-VH can give us an insight not only into the extent of plaque, but also on the extent of necrotic core within and beyond the intended stenting segment. In this context, studying 24 patients in whom 26 stented segments using IVUS-VH were assessed, no stent thrombosis was observed, where necrotic core rich areas were left unstented [40]. Ramcharitar et al. presented a first clinical case of a patients with a no culprit IVUS-VH derived thin capped fibroatheroma, successful treated with PCI, with a good 6-month angiographic follow-up [41]. A large trial in which patients are randomized to IVUS-VH or angiography guided optimal coronary stenting is required to draw firm conclusions.

Regarding stent evaluation by IVUS-VH, distinction should be made between metallic stents and BVS. Kim et al. have previously shown that metallic stents eluting sirolimus and paclitaxel introduce artifacts in IVUS-VH images, that interfere with the classification of plaque behind the struts [42]. Normally struts of drug-eluting stents appear as dense calcium, surrounded by a red halo. Although the BVS is made of non metallic materials, it was also recognized by IVUS-VH software as dense calcium and necrotic core. Moreover, for this scaffold, the presence of "pseudo" dense calcium and necrotic core could be used as quantitative surrogate for the presence of the polymeric material of the BVS and may help to evaluate the bioresorption process during follow-up [7, 8, 43, 44]. Garcia-Garcia et al. have already shown in a sub-study of ABSORB cohort A trial that polymeric struts are identified with radiofrequency backscattering signal as calcific structures and that the ability of IVUS-VH to recognize polymeric struts is important not only to study imaging of the BVS post-implantation, but also to potentially follow the mechanical support or bioresorption process [7] (Fig. 1).

Sarno et al. analyzing IVUS-VH plaque characterization behind the first generation BVS found a reduction in necrotic core component between 6-month and 2 year follow-up, probably related to a synergistic effect of the bioresorption process and the anti-inflammatory action of everolimus [8].

Since the stent's struts are recognized by IVUS$\mathrm{VH}$ as dense calcium and necrotic core, however, careful evaluation of exclusively the plaque behind the stent should be done to avoid any misclassification of the actual tissue [8, 42]. At the same time to assess in-stent restenosis in metallic stents, IVUS-VH has potential limitations in lack of validation of the technique in this context.

Kubo et al., analyzing the long-term effects of DES and of BMS on coronary arterial wall morphology by IVUS-VH, have shown that DES-treated lesions had a greater frequency of unstable lesion morphometry at follow-up compared to BMS [6]. In particular assessing the total amount of the four color-VH components including also the contribution of the stent, they found that DES-treated lesions showed at follow-up a significantly higher incidence of necrotic core abutting the lumen compared to BMS-treated lesions, although there was not a significant difference in necrotic core mean area between the two groups. This was due to a suppression of the protective neointimal hyperplasia layer in DES compared with BMS. Aoki et al. have demonstrated in vivo that plaque volume behind the metallic stent eluting sirolimus increases slightly at 4 months follow-up and then significantly decreases at 4 years follow-up compared to post-implantation and that 
Fig. 1 Serial changes in the struts of BVS 1.0 as assessed by IVUS-VH. On the left hand side, grayscale IVUS images are shown at pre-, post-implantation and 6-month follow-up. On the right hand side, their corresponding color code $\mathrm{VH}$ images are depicted. Of note, increased in dense calcium (white) and necrotic core (red) are noted after BVS implantation. At follow-up, a change in the BVS' strut appearance in grayscale IVUS is noted and decreases in the dense calcium and necrotic core contents in IVUS-VH

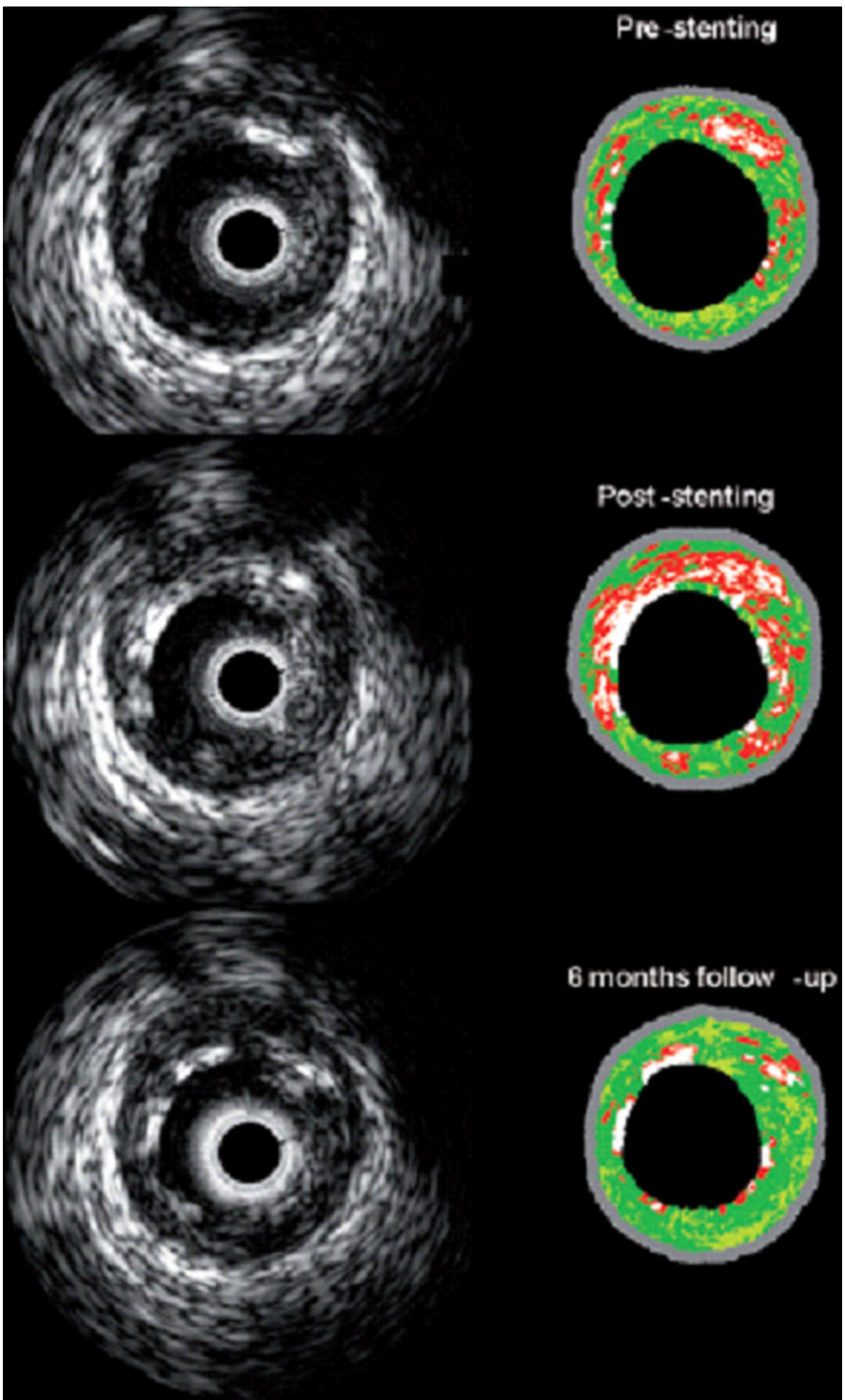

change in echogenicity is suggestive of a change in plaque composition [45]. He also documented a significantly increase of plaque area outside metallic stent eluting paclitaxel at 6 months, with its regression at 2 years [23]. In these situations, it would be interesting to know what type of tissue contributed to 
this process and, from a quantitative point, to develop a third (stent) contour in the VH software (VIAS or $\mathrm{pcVH}$ ) in order to characterize selectively intimal hyperplasia and peri-stent tissue.

\section{Edge effects assessed by IVUS-VH}

Based on previous pathological studies, we hypothesized that the tissue associated with increase of plaque at the edges of the paclitaxel stent is mainly fibro-fatty tissue as assessed by IVUS-VH. Fibrofatty, in IVUS-VH, has been described as loosely packed bundles of collagen fibers with regions of lipid deposition and extracellular matrix without necrotic areas [39].

In the BETAX study $[24,46]$ patients (26 paclitaxel eluting stents) were studied. Serial expansive vascular remodeling was observed at the proximal and distal edges of the stent to accommodate fibrofatty and fibrous tissue growth. More specifically, proximal and distal segments were divided into five sub-segments of $1-\mathrm{mm}$. In the first two subsegments adjacent to the proximal edge of the stent, the vessel wall grew to compensate the plaque growth without affecting the lumen size. In the following three sub-segments, overcompensation (vessel wall increased more than plaque size) was observed. Consequently, the lumen size increased. At the distal edge, overcompensation was observed in all five subsegments, followed by an increase in lumen size. In summary, proximal and distal growth patterns were characterized by an increase in fibrofatty tissue $(P<0.001$ and $P<0.001$, respectively), decrease in necrotic core $(P=0.014$ and $P<0.001$, respectively), and decrease in dense calcium content $(P<0.001$ and $P<0.001$ respectively).

\section{iMAP-IVUS}

Unlike VH, iMap $^{\mathrm{TM}}$ IVUS (iMap, $40 \mathrm{MHz}$, mechanical-type transducer, 3.2 F Atlantis, Boston Scientific Corporation) uses a $40 \mathrm{MHz}$ single rotational transducer on a drive shaft and can acquire radiofrequency data continuously. For the tissue characterization, it uses a pattern recognition algorithm on the spectra that were obtained from a fast fourier transformation and a histology-derived database [47]. iMap has higher resolution, but displays specific artifacts such as non-uniform rotational distortion because it is a rotational catheter.

Shin et al., compared in vivo iMap and VH for the detection of coronary atherosclerotic plaques and stent's struts [48]. iMap showed all struts of a stent separately where VH showed sometimes a continuum in between struts. Moreover, VH tended to overestimate metallic stent struts, and iMap showed thinner stent thickness than $\mathrm{VH}$. Also, there was peri-stent and calcium necrotic core halo in $\mathrm{VH}$ that was not seen in iMap (Fig. 2). For these reasons, iMap may be a good technique for metallic stent studies with serial evaluation because it provides clearer images of stent struts and does not produce necrotic tissue around stent struts. The stent struts were misclassified and overestimated as calcium in $\mathrm{VH}$. $\mathrm{VH}$ also increases the estimated amount of necrotic tissue in stented lesion, which is shown as necrotic core around the stent that is an artifact and should not be interpreted as peri-strut inflammation or necrotic core [42]. For these reasons, the study of overtime modification of dense calcium and necrotic core content of the plaque behind stent struts, especially behind the polymeric struts of a BVS, could be more accurate by iMAP.

\section{Palpography}

Palpography is an IVUS-based technique that assesses the local mechanical properties of the coronary plaque. The technique measures the relative displacements of backscattered radiofrequency signals, recorded during IVUS acquisition, at two different blood pressure levels to detect differences in deformability or strain of various plaque components. As such, lipid-rich plaques will deform more and thus show a higher strain value compared with calcified or fibrous plaques [49]. Palpography data are acquired during a conventional IVUS pullback with a commercially available catheter $(20 \mathrm{MHz}$ Jovus Avanar, Volcano, Rancho Cordova, California). Plaque strain values are classified according to the Rotterdam classification (ROC) score in a range from I to IV (ROC I: $0-0.6 \%$; ROC II: 0.6 to $<0.9 \%$; ROC III: 0.9 to $<1.2 \%$; ROC IV: $>1.2 \%$ ) [50].

Palpography has been used to evaluate postimplantation effect of BVS. In a sub-study of the ABSORB A trial, the mean cumulative strain values 
Fig. 2 There is no necrotic core around stent struts by iMap, which shows clearly separated stent struts (a). Metallic stent struts appear as confluent dense calcium surrounded by a necrotic core in $\mathrm{VH}(\mathbf{b})$
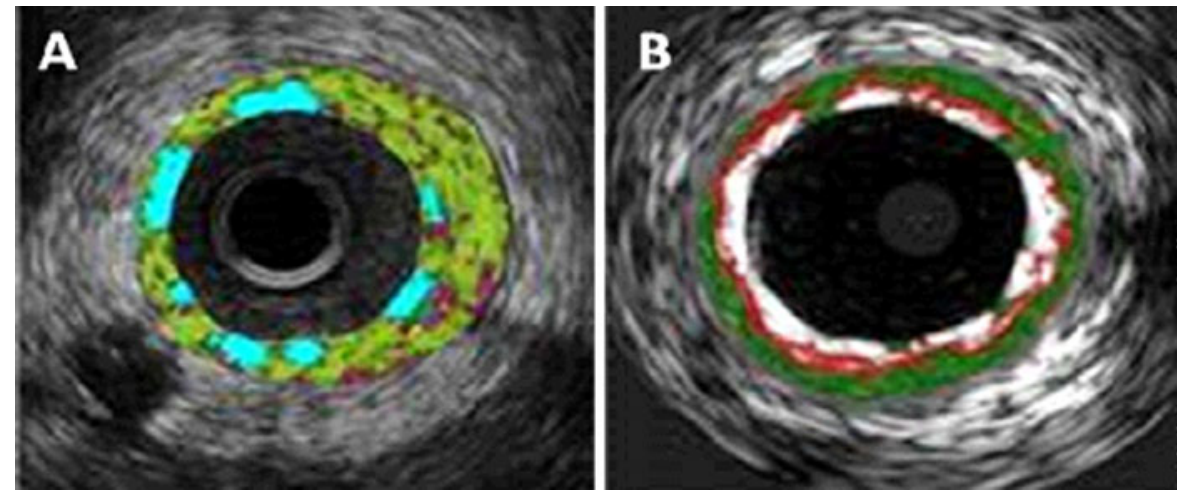

in all frames with ROC I-IV scores changed from $0.50 \pm 0.27-0.20 \pm 0.10 \% \quad(P=0.0034) \quad$ [7]. In 6 months follow-up the mean cumulative strain values in all frames with ROC I-IV scores increased significantly, compared to post-implantation. Deployment of a stent against the vessel wall undoubtedly modifies the physical properties of the endoluminal surface. With BVS, the reduction of the high strain values immediately post-BVS implantation may be due to a real decrease in deformability of the scaffolded vessel wall. Alternatively, it may also reflect an ultrasonic artifact, namely the incapability of palpography to measure intrinsic changes in strain of the vessel due to the acoustic properties of the BVS struts preventing a proper propagation of radiofrequency signal behind them. The following increase of the strain values at follow-up may be an indirect indicator of modification in strut structure of BVS [7, 43, 44].

New frontiers: optical coherence tomography

With the increasing diffusion of the Optical Coherence Tomography (OCT), it is important to consider its differences with the ultrasound imaging modalities in guided coronary intervention. OCT images provide a clear depiction of the boundaries between lumen and vessel. OCT's ability to penetrate and delineate calcium in the vessel wall makes it well suited to guide complex interventional strategies in vessels with superficial calcification [51]. OCT has also higher sensitivity compared with IVUS for detection of malapposed strut [52]. Plaque protrusion and stentedge dissection are other common interventionrelated parameters readily visible on OCT images, although their clinical impact remains to be assessed.

\section{Conclusions}

IVUS is the most widely available tool for assessing most features of stent implantation. Evaluation of optimal stent deployment, good stent apposition and coverage of the plaque are very important topics to consider when a PCI with DES is performed and IVUS is helpful to the operator. Probably IVUS should be performed in all patients with DES failure to better understand the pathophysiology. IVUS-VH can add complementary information to study the edge effects in metallic stents and can give insights into the temporal compositional changes in bioresorbable scaffold studies. iMAP probably is a better method to obtain information about changes in bioresorbable scaffold, due to less artifacts in stent struts misreading; however, there are no data on this.

\section{References}

1. Das P, Meredith I (2007) Role of intravascular ultrasound in unprotected left main percutaneous coronary intervention. Expert Rev Cardiovasc Ther 5:81-89

2. Smith SC Jr, Feldman TE, Hirshfeld JW Jr et al (2006) ACC/ AHA/SCAI 2005 guideline update for percutaneous coronary intervention-summary article: a report of the American College of Cardiology/American Heart Association Task Force on Practice Guidelines (ACC/AHA/SCAI Writing Committee to Update the 2001 Guidelines for Percutaneous Coronary Intervention). Circulation 113:156-75

3. Nair A, Kuban BD, Tuzcu EM, Schoenhagen P, Nissen SE, Vince DG (2002) Coronary plaque classification with intravascular ultrasound radiofrequency data analysis. Circulation 106:2200-2206

4. Nair A, Margolis MP, Kuban BD, Vince DG (2007) Automated coronary plaque characterisation with intravascular ultrasound backscatter: ex vivo validation. EuroIntervention 3:113-120 
5. Ramcharitar S, Garcia-Garcia HM, Nakazawa G et al (2007) Ultrasonic and pathological evidence of a neointimal plaque rupture in patients with bare metal stents. EuroIntervention 3:290-291

6. Kubo T, Maehara A, Mintz GS et al (2010) Analysis of the long-term effects of drug-eluting stents on coronary arterial wall morphology as assessed by virtual histology intravascular ultrasound. Am Heart J 159:271-277

7. Garcia-Garcia HM, Gonzalo N, Pawar R et al (2009) Assessment of the absorption process following bioabsorbable everolimus-eluting stent implantation: temporal changes in strain values and tissue composition using intravascular ultrasound radiofrequency data analysis. A substudy of the ABSORB clinical trial. EuroIntervention 4:443-448

8. Sarno G, Onuma Y, Garcia HM et al (2010) IVUS radiofrequency analysis in the evaluation of the polymeric struts of the bioabsorbable everolimus-eluting device during the bioabsorption process. Catheter Cardiovasc Interv 75:914918

9. Mintz GS (2007) Features and parameters of drug-eluting stent deployment discoverable by intravascular ultrasound. Am J Cardiol 100:26M-35M

10. Moses JW, Dangas G, Mehran R, Mintz GS (2008) Drugeluting stents in the real world: how intravascular ultrasound can improve clinical outcome. Am J Cardiol 102: 24J-28J

11. Roy P, Steinberg DH, Sushinsky SJ et al (2008) The potential clinical utility of intravascular ultrasound guidance in patients undergoing percutaneous coronary intervention with drug-eluting stents. Eur Heart J 29:1851-1857

12. Costa MA, Angiolillo DJ, Tannenbaum M et al (2008) Impact of stent deployment procedural factors on longterm effectiveness and safety of sirolimus-eluting stents (final results of the multicenter prospective STLLR trial). Am J Cardiol 101:1704-1711

13. de Jaegere P, Mudra H, Figulla H et al (1998) Intravascular ultrasound-guided optimized stent deployment. Immediate and 6 months clinical and angiographic results from the multicenter ultrasound stenting in coronaries study (MUSIC Study). Eur Heart J 19:1214-1223

14. Fujii K, Carlier SG, Mintz GS et al (2005) Stent underexpansion and residual reference segment stenosis are related to stent thrombosis after sirolimus-eluting stent implantation: an intravascular ultrasound study. J Am Coll Cardiol 45:995-998

15. Okabe T, Mintz GS, Buch AN et al (2007) Intravascular ultrasound parameters associated with stent thrombosis after drug-eluting stent deployment. Am J Cardiol 100:615-620

16. Sonoda S, Morino Y, Ako J et al (2004) Impact of final stent dimensions on long-term results following sirolimus-eluting stent implantation: serial intravascular ultrasound analysis from the sirius trial. J Am Coll Cardiol 43:1959-1963

17. Hong MK, Mintz GS, Lee CW et al (2006) Intravascular ultrasound predictors of angiographic restenosis after sirolimus-eluting stent implantation. Eur Heart J 27:1305-1310

18. Sakurai R, Ako J, Morino Y et al (2005) Predictors of edge stenosis following sirolimus-eluting stent deployment (a quantitative intravascular ultrasound analysis from the SIRIUS trial). Am J Cardiol 96:1251-1253
19. Wentzel JJ, Gijsen FJ, Stergiopulos N, Serruys PW, Slager CJ, Krams R (2003) Shear stress, vascular remodeling and neointimal formation. J Biomech 36:681-688

20. Serruys PW, Degertekin M, Tanabe K et al (2004) Vascular responses at proximal and distal edges of paclitaxeleluting stents: serial intravascular ultrasound analysis from the TAXUS II trial. Circulation 109:627-633

21. Weissman NJ, Koglin J, Cox DA et al (2005) Polymerbased paclitaxel-eluting stents reduce in-stent neointimal tissue proliferation: a serial volumetric intravascular ultrasound analysis from the TAXUS-IV trial. J Am Coll Cardiol 45:1201-1205

22. Sakurai R, Hongo Y, Yamasaki M et al (2007) Detailed intravascular ultrasound analysis of Zotarolimus-eluting phosphorylcholine-coated cobalt-chromium alloy stent in de novo coronary lesions (results from the ENDEAVOR II trial). Am J Cardiol 100:818-823

23. Aoki J, Colombo A, Dudek D et al (2005) Peristent remodeling and neointimal suppression 2 years after polymer-based, paclitaxel-eluting stent implantation: insights from serial intravascular ultrasound analysis in the TAXUS II study. Circulation 112:3876-3883

24. Tanabe K, Serruys PW, Degertekin M et al (2005) Incomplete stent apposition after implantation of paclitaxel-eluting stents or bare metal stents: insights from the randomized TAXUS II trial. Circulation 111:900-905

25. Ako J, Morino Y, Honda Y et al (2005) Late incomplete stent apposition after sirolimus-eluting stent implantation: a serial intravascular ultrasound analysis. J Am Coll Cardiol 46:1002-1005

26. Kimura M, Mintz GS, Carlier S et al (2006) Outcome after acute incomplete sirolimus-eluting stent apposition as assessed by serial intravascular ultrasound. Am J Cardiol 98:436-442

27. Hong MK, Mintz GS, Lee CW et al (2006) Late stent malapposition after drug-eluting stent implantation: an intravascular ultrasound analysis with long-term follow-up. Circulation 113:414-419

28. Serruys PW, Ruygrok P, Neuzner J et al (2006) A randomised comparison of an everolimus-eluting coronary stent with a paclitaxel-eluting coronary stent:the SPIRIT II trial. EuroIntervention 2:286-294

29. Escolar E, Mintz GS, Popma J et al (2007) Meta-analysis of angiographic versus intravascular ultrasound parameters of drug-eluting stent efficacy (from TAXUS IV, V, and VI). Am J Cardiol 100:621-626

30. Cook S, Wenaweser P, Togni M et al (2007) Incomplete stent apposition and very late stent thrombosis after drugeluting stent implantation. Circulation 115:2426-2434

31. de Ribamar Costa J, Mintz GS Jr, Carlier SG Jr et al (2007) Intravascular ultrasound assessment of drug-eluting stent expansion. Am Heart J 153:297-303

32. Shaikh F, Maddikunta R, Djelmami-Hani M, Solis J, Allaqaband S, Bajwa T (2008) Stent fracture, an incidental finding or a significant marker of clinical in-stent restenosis? Catheter Cardiovasc Interv 71:614-618

33. Yamada KP, Koizumi T, Yamaguchi H et al (2008) Serial angiographic and intravascular ultrasound analysis of late stent strut fracture of sirolimus-eluting stents in native coronary arteries. Int J Cardiol 130:255-259 
34. Doi H, Maehara A, Mintz GS et al (2009) Classification and potential mechanisms of intravascular ultrasound patterns of stent fracture. Am J Cardiol 103:818-823

35. Farb A, Kolodgie FD, Hwang JY et al (2004) Extracellular matrix changes in stented human coronary arteries. Circulation 110:940-947

36. Finn AV, Nakazawa G, Joner M et al (2007) Vascular responses to drug eluting stents: importance of delayed healing. Arterioscler Thromb Vasc Biol 27:1500-1510

37. Nasu K, Tsuchikane E, Katoh O et al (2006) Accuracy of in vivo coronary plaque morphology assessment: a validation study of in vivo virtual histology compared with in vitro histopathology. J Am Coll Cardiol 47:2405-2412

38. Farb A, Burke AP, Kolodgie FD, Virmani R (2003) Pathological mechanisms of fatal late coronary stent thrombosis in humans. Circulation 108:1701-1706

39. MP NairA, Kuban BD, Vince DG (2007) Automated coronary plaque characterization with intravascular ultrasound backscatter: ex vivo validation. Eurointervention 3:113130

40. Garcia-Garcia HM, Goedhart D, Serruys PW (2007) Relation of plaque size to necrotic core in the three major coronary arteries in patients with acute coronary syndrome as determined by intravascular ultrasonic imaging radiofrequency. Am J Cardiol 99:790-792

41. Ramcharitar S, Gonzalo N, van Geuns RJ et al (2009) First case of stenting of a vulnerable plaque in the SECRITT I trial-the dawn of a new era? Nat Rev Cardiol 6:374-378

42. Kim SW, Mintz GS, Hong YJ et al (2008) The virtual histology intravascular ultrasound appearance of newly placed drug-eluting stents. Am J Cardiol 102:1182-1186

43. Serruys PW, Ormiston JA, Onuma Y et al (2009) A bioabsorbable everolimus-eluting coronary stent system (ABSORB): 2-year outcomes and results from multiple imaging methods. Lancet 373:897-910

44. Ormiston JA, Serruys PW, Regar E et al (2008) A bioabsorbable everolimus-eluting coronary stent system for patients with single de novo coronary artery lesions
(ABSORB): a prospective open-label trial. Lancet 371: 899-907

45. Aoki J, Abizaid AC, Serruys PW et al (2005) Evaluation of four-year coronary artery response after sirolimus-eluting stent implantation using serial quantitative intravascular ultrasound and computer-assisted grayscale value analysis for plaque composition in event-free patients. J Am Coll Cardiol 46:1670-1676

46. Garcia-Garcia HM, Gonzalo N, Tanimoto S, Meliga E, de Jaegere P, Serruys PW (2008) Characterization of edge effects with paclitaxel-eluting stents using serial intravascular ultrasound radiofrequency data analysis: the BETAX (BEside TAXus) Study. Rev Esp Cardiol 61:1013-1019

47. Sathyanarayana S, Carlier S, Li W, Thomas L (2009) Characterisation of atherosclerotic plaque by spectral similarity of radiofrequency intravascular ultrasound signals. EuroIntervention 5:133-139

48. Shin ES, Garcia Garcia HM, Lighthart J, et al (2010) In Vivo Findings of Tissue Characteristics using iMap ${ }^{\mathrm{TM}}$ IVUS and Virtual Histology ${ }^{\mathrm{TM}}$ IVUS EuroInterv In press 2010

49. de Korte CL, Sierevogel MJ, Mastik F et al (2002) Identification of atherosclerotic plaque components with intravascular ultrasound elastography in vivo: a Yucatan pig study. Circulation 105:1627-1630

50. Van Mieghem CA, McFadden EP, de Feyter PJ et al (2006) Noninvasive detection of subclinical coronary atherosclerosis coupled with assessment of changes in plaque characteristics using novel invasive imaging modalities: the Integrated Biomarker and Imaging Study (IBIS). J Am Coll Cardiol 47:1134-1142

51. Bezerra HG, Costa MA, Guagliumi G, Rollins AM, Simon DI (2009) Intracoronary optical coherence tomography: a comprehensive review clinical and research applications. JACC Cardiovasc Interv 2:1035-1046

52. Bouma BE, Tearney GJ, Yabushita H et al (2003) Evaluation of intracoronary stenting by intravascular optical coherence tomography. Heart 89:317-320 